Diabetologe 2018 $\cdot 14: 212-213$

https://doi.org/10.1007/s11428-018-0344-9

Online publiziert: 17. Mai 2018

(c) Springer Medizin Verlag $\mathrm{GmbH}$, ein Teil von Springer Nature 2018

CrossMark

\section{P. Achenbach}

Institut für Diabetesforschung, Helmholtz Zentrum München, München, Deutschland
Typ-1-Diabetes ist mit etwa 30.000 erkrankten Kindern und Jugendlichen die häufigste pädiatrische Stoffwechselerkrankung in Deutschland - mit steigender Tendenz. Seit Jahren wird weltweit eine dramatische Zunahme der Neuerkrankungsfälle beobachtet. Dies betrifft insbesondere die jüngsten Altersgruppen.

Etwa ein Drittel aller Kinder erleiden bei klinischer Manifestation des Typ-1Diabetes eine schwere Stoffwechselentgleisung (Ketoazidose) als Konsequenz einer zu späten Diagnosestellung. Das muss nicht sein! Ein frühes Erkennen und Behandeln der Erkrankung sind möglich und dringend erforderlich, um die gegenwärtige Situation zu verbessern.

》) Ziel sollte sein, möglichst alle Personen zu identifizieren, die ein Frühstadium entwickeln

Typ-1-Diabetes ist eine Autoimmunerkrankung, die durch die Zerstörung der insulinproduzierenden Zellen in der Bauchspeicheldrüse entsteht. Die Krankheitsentwicklung durchläuft asymptomatische Frühstadien, die mit immunologischen Tests sehr gut diagnostizierbar sind. Das Risiko, bereits in der Kindheit ein Frühstadium des Typ-1-Diabetes zu entwickeln, ist ebenfalls sehr gut durch neue genetische Testverfahren bestimmbar. Liegt ein Frühstadium vor, können Betroffene geschult und die Progression zur klinisch manifesten Erkrankung mit metabolischen Tests im Verlauf beobachtet und eingeschätzt werden. Dadurch kann bei Bedarf frühzeitig mit einer Insulintherapie begonnen werden, um Stoffwechselentgleisungen $\mathrm{zu}$ verhindern. Zudem ist die Früherkennung eine
Voraussetzung, um präventive Behandlungen erfolgreich zu etablieren.

Bei Verwandten von Patienten mit Typ-1-Diabetes werden Früherkennungsuntersuchungen schon seit vielen Jahren im Rahmen von Studien praktiziert, also in einer Personengruppe mit erhöhtem familiärem Erkrankungsrisiko. Allerdings haben nur etwa $10 \%$ der Patienten mit Typ-1-Diabetes einen ebenfalls erkrankten Verwandten ersten Grades, und weitere 10\% haben einen von dieser Erkrankung betroffenen Verwandten zweiten Grades. Das heißt, dass für mindestens $80 \%$ der Betroffenen bisher keine Möglichkeit bestand, an Früherkennungsuntersuchungen teilzunehmen. Gleichzeitig wurde diese Personengruppe fast vollständig von der Teilnahme an Präventionsmaßnahmen ausgeschlossen. Ein wesentlicher Grund für das bisherige Vorgehen ist die vergleichsweise geringere Prävalenz der Erkrankung bei Personen aus nicht betroffenen Familien. Die Wahrscheinlichkeit, ein Frühstadium des Typ-1Diabetes bei Kindern aus der Allgemeinbevölkerung $\mathrm{zu}$ diagnostizieren, ist etwa 10 -fach niedriger als bei Kindern mit betroffenen Verwandten. Dabei ist jedoch $\mathrm{zu}$ betonen, dass es für die Progression vom Frühstadium bis zur klinischen Erkrankung unerheblich ist, ob eine familiäre Belastung vorliegt oder nicht. Ziel sollte deshalb sein, möglichst alle Personen $\mathrm{zu}$ identifizieren, die ein Frühstadium entwickeln. Um eine effektive Senkung der Inzidenz des Typ1-Diabetes sowie der Komplikationsrate bei klinischer Manifestation zu erzielen, müssen bevölkerungsweite Untersuchungen erfolgen, damit die Mehrheit der Betroffenen die Chance erhält, von Präventionsmaßnahmen zu profitieren.
Zweifellos ist ein Screening auf Populationsebene eine sehr große Herausforderung. Projekte wie Fr1da (Typ-1Diabetes: Früh erkennen - Früh gut behandeln) und Fr1dolin (Früherkennung von Typ-1-Diabetes und Hypercholesterinämie in Niedersachsen) zur Frühdiagnose des Typ-1-Diabetes bei Kindern in Deutschland (s. Beiträge von Winkler et al. und Böttcher et al.) haben deshalb eine weltweite Vorreiterrolle und werden mittlerweile auch in anderen Ländern begonnen; ein Beispiel ist ASK („autoimmunity screening for kids") in Colorado. Für die Durchführung großer Studien zur Primärprävention ist sogar ein genetisches Screening von Hunderttausenden an Neugeborenen erforderlich, um Kinder mit einem erhöhten Risiko für Typ-1-Diabetes zu identifizieren. Dies erfordert internationale Zusammenarbeit; GPPAD („Global Platform for the Prevention of Autoimmune Diabetes") ist dafür beispielhaft (s. Beitrag von Ziegler et al.). Dass ein genetisches Screening von Neugeborenen in Deutschland erfolgreich durchführbar ist, zeigen die Erfahrungen der Freder1k-Pilotstudie in Sachsen (s. Beitrag von Hommel).

Natürlich verbleiben noch offene Fragen, die beantwortet werden müssen. Erforderlich ist eine eingehende Bewertung hinsichtlich Nutzen/Risiko, Durchführbarkeit, Akzeptanz und Effektivität der Früherkennungsuntersuchungen (s. Beiträge von Lange und Beyerlein u. Jolink). Bislang existieren auch noch keine weitreichenden Erfahrungen oder Leitlinien zur Behandlung des Typ1-Diabetes im Frühstadium, was eine neue therapeutische Herausforderung darstellt (s. Beitrag von Warncke et al.). Die präventive Behandlung mit oralem Insulin wird gegenwärtig in mehreren 
klinischen Studien geprüft (s. Beitrag von Achenbach), bei Kindern mit einem asymptomatischen Frühstadium des Typ-1-Diabetes (Fr1da-Insulin-Interventions-Studie) sowie Kleinkindern mit einem genetischen Risiko für diese Erkrankung (POInT [ „primary oral insulin trial"]), ist aber noch keine gesichert wirksame Therapie. Neue Behandlungsformen waren bereits in präklinischen Studien erfolgreich und könnten in $\mathrm{Zu}$ kunft auch beim Menschen Anwendung finden (s. Beitrag von Serr u. Daniel).

Bemerkenswert ist die Tatsache, dass die nicht unerheblichen Kosten der genannten Projekte zu großen Teilen von 2 nichtkommerziellen Organisationen aus den USA getragen werden, dem „Leona M. \& Harry B. Helmsley Charitable Trust “ und der JDRF „Juvenile Diabetes Research Foundation“. Dies unterstreicht die Bedeutung und innovative Rolle der Typ-1-Diabetes-Forschung in Deutschland und Europa.

\section{》) Die Vision ist, die Entstehung von Typ-1-Diabetes zu verhindern}

Die Vision ist, die Zunahme von Diabetes im Kindesalter einzudämmen und die Entstehung von Typ-1-Diabetes zu verhindern. Die flächendeckende Einführung einer Risikobestimmung für Typ1-Diabetes bei Neugeborenen und einer Frühdiagnose der Erkrankung im Kindesalter soll den Weg zu neuen präventiven, personalisierten Behandlungen eröffnen. Prävention und Frühdiagnose sollen als feste Komponenten in die Versorgung des Diabetes im Kindesalter verankert werden. Die Schaffung nachhaltiger Organisationsstrukturen und Plattformen soll eine breit angelegte klinische Forschung auf dem Gebiet des Diabetes im Kindesalter ermöglichen und könnte auch zur Behandlung weiterer chronischer Stoffwechselerkrankungen im Kindesalter dienen. Die Realisierung dieser Vision braucht Unterstützung; gemeinsam können wir die Weichen stellen, in eine Zukunft ohne Typ-1-Diabetes bei Kindern.

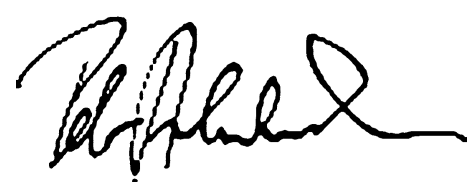

PD Dr. P. Achenbach

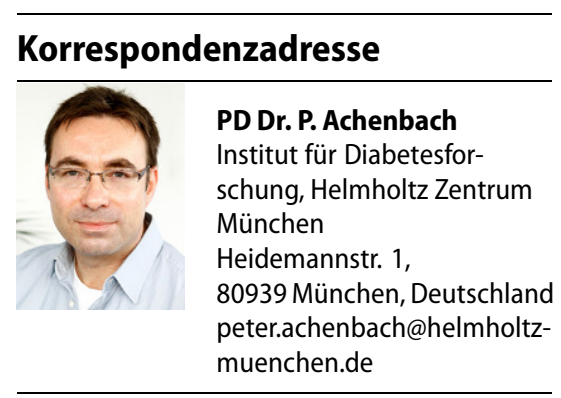

Interessenkonflikt. P. Achenbach gibt an, dass kein Interessenkonflikt besteht.

\section{Weniger Amputationen bei Menschen mit Diabetes}

Beim diabetischen Fußsyndrom kommt es zu schlecht heilenden Wunden im Fußbereich. Diese Wunden können Amputationen nach sich ziehen. Wie häufig Amputationen der unteren Extremität bei Personen mit Diabetes in Deutschland auftraten - und wie sich diese Häufigkeit im Laufe der Jahre verändert hat, hat ein Team von Wissenschaftler/Innen am Deutschen Diabetes-Zentrum (DDZ) in Kooperation mit gesetzlichen Krankenkassen, AOK, BARMER und Betriebskrankenkassen, klinischen Partnern und den Universitäten Köln und Oldenburg, mittels Daten von 34 Millionen gesetzlich Versicherten untersucht. Sie fanden heraus, dass die Zahl der Amputationen bei Menschen mit Diabetes im Laufe der Jahre zurückgegangen ist. In ihrer Analyse haben die Wissenschaftler unterschieden zwischen größeren Amputationen der unteren Extremität, das heißt, solchen oberhalb des Sprunggelenks und kleineren, das heißt durch oder unterhalb des Sprunggelenks. Die Häufigkeit für Amputationen der unteren Extremität wurde dabei für den Zeitraum von 2008 bis 2012 für Personen mit und ohne Diabetes untersucht. Bei rund 11 Prozent aller Versicherten in der Studie wurde Typ-1- oder Typ-2-Diabetes diagnostiziert. Insgesamt musste von 2008 bis 2012 bei 108.208 Teilnehmern mindestens eine Amputation der unteren Extremität vorgenommen werden. „Von diesen litten rund zwei Drittel an Diabetes", erklärt Prof. Dr. Dr. Andrea Icks, Direktorin des Instituts für Gesundheitsökonomie und Versorgungsforschung am DDZ. Im Durchschnitt waren die Patienten zum Zeitpunkt ihrer ersten Amputation der unteren Extremität 73 Jahre alt. Bei Menschen mit Diabetes waren im Laufe der Jahre sowohl die Zahl der größeren als auch die der kleineren Amputationen der unteren Extremität rückläufig.

\section{Originalpublikation:}

Claessen H et al. (2018) Lower-extremity amputations in people with and without diabetes in Germany. Clinical Epidemiology. 10:475-488.

Quelle: Deutsches Diabetes-Zentrum, www.ddz.uni-duesseldorf.de 